have vaginal sex to not wash their hands properly $(19.4 \%$ vs. $3.0 \%, \mathrm{p}<0.03)$ and not wear a mask $(30.8 \%$ vs. $10.6 \%$, $\mathrm{p}<0.02)$; there was a trend for them to not physically distance $(28.2 \%$ vs. $16.7 \%, \mathrm{p}<0.17)$ and there was no association for not following stay-at-home orders $(28.2 \%$ vs. $22.7 \%$, $\mathrm{p}<0.54)$. Age, access to masks, and perception that maskwearing could subject them to discrimination were not associated with mask-wearing ( $\mathrm{p}>0.40)$.

Conclusion Overall, adherence to disease prevention behaviors was high. Non-adherent behaviors such as condomless sex, lack of mask-wearing and lack of hand-washing were associated. Men with one non-adherent behavior may benefit from counseling in other potentially non-adherent behaviors.

\section{P405 IDENTIFYING SUBGROUPS AT HIGHER RISK OF INFECTIOUS SYPHILIS IN MAJOR AUSTRALIAN CITIES: ANALYSIS OF NATIONAL SENTINEL SURVEILLANCE DATA 2011-2018}

\begin{abstract}
${ }^{1,2} \mathrm{~A}$ Carter ${ }^{*},{ }^{1} \mathrm{H}$ McManus, ${ }^{1} \mathrm{~T}$ Vickers, ${ }^{3} \mathrm{~J}$ Asselin, ${ }^{4,5,6} \mathrm{E}$ Chow, ${ }^{4,5} \mathrm{M}$ Chen, ${ }^{4,5} \mathrm{C}$ Fairley, ${ }^{1,7} \mathrm{C}$ Bourne, ${ }^{7,8} \mathrm{~A}$ McNulty, ${ }^{1,9} \mathrm{P}$ Read, ${ }^{10} \mathrm{~N}$ Ryder, ${ }^{11,12} \mathrm{~J} \mathrm{McCloskey,}{ }^{13} \mathrm{C}$ Carmody, ${ }^{3,14,15} \mathrm{M}$ Stoové, ${ }^{3,14,16} \mathrm{M}$ Hellard, 'B Donovan, ' $\mathrm{R}$ Guy, ${ }^{1,3} \mathrm{ACC}$ CSS Collaboration. ${ }^{1}$ Kirby Institute, UNSW Sydney, Sydney, Australia; ${ }^{2}$ Faculty of Health Sciences, Simon Fraser University, Burnaby, Canada; ${ }^{3}$ Burnet Institute, Melbourne, Australia; ${ }^{4}$ Melbourne Sexual Health Centre, Alfred Health, Melbourne, Australia; ${ }^{5}$ Central Clinical School, Monash University, Melbourne, Australia; ${ }^{6}$ Centre for Epidemiology and Biostatistics, Melbourne School of Population and Global Health, The University of Melbourne, Melbourne, Australia; ${ }^{7}$ Sydney Sexual Health Centre, Sydney Hospital, Sydney, Australia; ${ }^{8}$ School of Public Health and Community Medicine, UNSW Sydney, Sydney, Australia; ${ }^{9}$ Kirketon Road Centre, Sydney, Australia; ${ }^{10}$ Hunter New England Clinic, Sydney, Australia; ${ }^{11}$ Royal Perth Hospital Sexual Health Clinic, Perth, Australia; ${ }^{12}$ Division of Infection and Immunity, The University of Western Australia, Perth, Australia; ${ }^{13}$ Liverpool Sexual Health Clinic, Sydney, Australia; ${ }^{14}$ School of Public Health and Preventive Medicine, Monash University, Melbourne, Australia; ${ }^{15}$ School of Psychology and Public Health, La Trobe University, Melbourne, Australia; ${ }^{16}$ Department of Infectious Diseases, Alfred Health and Monash University, Melbourne, Australia
\end{abstract}

\subsection{6/sextrans-2021-sti.435}

Background In Australia, infectious syphilis notifications have increased 234\% in the past decade, from 1318 in 2008 to 4398 in 2017. Although historically concentrated among urban men who have sex with men and remote Indigenous communities, a rise in syphilis notifications among women in major cities and cases of congenital syphilis have been observed. We analysed trends in infectious syphilis positivity among women and heterosexual men in major Australian cities and identified associated risk factors.

Methods De-identified patient data were extracted from 34 sexual health clinics within a national sentinel surveillance network (2011-18). All women and heterosexual men $\geq 15$ years in major cities were included. Infectious syphilis positivity was defined as the proportion of attendees per 6-monthly calendar period with recorded syphilis testing who had recorded clinical diagnoses of infectious syphilis. Poisson regression determined annual trends in positivity and risk factors for infectious syphilis (rate ratios and 95\% CIs).

Results Of 100,230 patients attending the clinics, 50.8\% were female (of whom, 96.1\% were of childbearing age), $51.2 \%$ were aged 15-29 years, $1.7 \%$ were Indigenous and $7.4 \%$ were from culturally and linguistically diverse (CALD) backgrounds. Modelled positivity for infectious syphilis (2011-18) increased $85 \%$ in females from 2.41 (95\% CI:1.67-3.14) to 4.48 (95\%CI:3.53-5.43) per 1000 patients tested, and $76 \%$ in heterosexual males from 4.47 (95\%CI:3.44-5.49) to 7.87 (95\%CI:6.49-9.24). Factors associated with increased risk of infectious syphilis included: reporting a history of injecting drug use (RR:4.36;95\%CI:3.26-5.83), using condoms inconsistently in the past year (RR:2.30;95\%CI;1.10-4.79), being male (RR:1.80;95\%CI:1.51-2.14), Indigenous (RR:1.70;95\% CI:1.02-2.84), from CALD backgrounds (RR:1.90;95\% CI:1.46-2.46), and older (30-39 years: RR:1.33;95\%CI:1.08$1.65 ; 40-49$ years: RR:2.95;95\%CI:2.34-3.73; 50+ years: RR:3.59;95\%CI:2.81-4.57). Lower risk was observed in bisexual females (RR:0.53;95\%CI:0.30-0.94) and female sex workers (RR:0.43;95\%CI:0.29-0.63).

Conclusion Increasing syphilis in women and heterosexual men in major Australian cities requires enhanced prevention, including integration of sexual and reproductive health care into harm reduction programs.

\section{P407 MODEST MONETARY INCENTIVIZED PEER REFERRAL TO INCREASE CHLAMYDIA COMMUNITY-BASED TESTING AMONG YOUNG BLACK MEN WHO HAVE SEX WITH WOMEN}

G Gomes, M TatahMentan, C Ntambwe, G Scott, B Wise, M Palomares, N Schmidt, P Kissinger*. Tulane University School of Public Health and Tropical Medicine, 1440 Canal Street Suite 2004, USA

\subsection{6/sextrans-2021-sti.436}

Purpose The U.S. Centers for Disease Control and Prevention recommend screening young women for chlamydia but not young men. One reason young men have not been a focus for chlamydia screening is due to the perceived difficulty of accessing these men. Peers are among the most influential people in a young person's life. We hypothesized that incentivized peer referral (IPR) could assist in increasing enrollment in this screening study.

Methods The Check it Program in New Orleans is a community-based screening program for young Black men aged 15-24. Peer referral was monitored between 3/7/2018-11/28/2020. IPR started on $8 / 29 / 2020$ during which men enrolled in the parent study were offered $\$ 5$ for each peer they referred and were successfully enrolled in the study.

Results Of 1457 men enrolled in the study, 44 were enrolled after IPR was initiated. The percentage of men who said they were referred by a friend was higher in the post-IPR compared to the pre-IPR period $(40.9 \%$ vs. $20.0 \%, \mathrm{P}<0.001)$. Peer referral vouchers were claimed by 7 index men who successfully enrolled 9 men; two of the men referred 2 men. Those who said they heard about the study from a friend were: older ( 20.3 vs 19.9 years, $\mathrm{P}<0.02)$, more likely to live in a dorm $(21.7 \%$ vs $11.1 \%, \mathrm{P}<0.001)$, and to have prior chlamydia infection $(13.9 \%$, vs. $10.1 \%, \mathrm{P}=0.06)$. After adjusting for these factors, hearing about the study from a friend was higher in the post-IPR compared to the pre-IPR period with a 2.56 greater likelihood of enrolling (95\% C.I. 1.384.78, $\mathrm{p}<0.01)$

Conclusion Incentivized peer referral significantly increased study enrollment and can serve as a method for increasing screening rates in community-based chlamydia screening programs for young Black men who have sex with women. 